\title{
Improving Genomic Selection With Quantitative Trait Loci and Nonadditive Effects Revealed by Empirical Evidence in Maize
}

\section{OPEN ACCESS}

Edited by:

Ryo Fujimoto,

Kobe University, Japan

Reviewed by:

Akio Onogi,

National Agriculture and Food

Research Organization

(NARO), Japan

Manje Gowda,

The International Maize and Wheat Improvement Center

(CIMMYT), Kenya

${ }^{*}$ Correspondence:

Changling Huang

huangchangling@caas.cn

tThese authors have contributed equally to this work

Specialty section:

This article was submitted to Plant Breeding,

a section of the journal

Frontiers in Plant Science

Received: 06 June 2019

Accepted: 15 August 2019

Published: 18 September 2019

Citation:

Liu X, Wang H, Hu X, Li K, LiU Z, Wu Y and Huang C (2019) Improving Genomic Selection With Quantitative

Trait Loci and Nonadditive

Effects Revealed by Empirical

Evidence in Maize.

Front. Plant Sci. 10:1129.

doi: 10.3389/fpls.2019.01129

\section{Xiaogang Liut, Hongwu Wang', Xiaojiao Hu, Kun Li, Zhifang Liu, Yujin Wu and Changling Huang *}

\author{
Institute of Crop Science, National Key Facility of Crop Gene Resources and Genetic Improvement, Chinese Academy of \\ Agricultural Sciences, Beijing, China
}

Genomic selection (GS), a tool developed for molecular breeding, is used by plant breeders to improve breeding efficacy by shortening the breeding cycle and to facilitate the selection of candidate lines for creating hybrids without phenotyping in various environments. Association and linkage mapping have been widely used to explore and detect candidate genes in order to understand the genetic mechanisms of quantitative traits. In the current study, phenotypic and genotypic data from three experimental populations, including data on six agronomic traits (e.g., plant height, ear height, ear length, ear diameter, grain yield per plant, and hundred-kernel weight), were used to evaluate the effect of traitrelevant markers (TRMs) on prediction accuracy estimation. Integrating information from mapping into a statistical model can efficiently improve prediction performance compared with using stochastically selected markers to perform GS. The prediction accuracy can reach plateau when a total of 500-1,000 TRMs are utilized in GS. The prediction accuracy can be significantly enhanced by including nonadditive effects and TRMs in the GS model when genotypic data with high proportions of heterozygous alleles and complex agronomic traits with high proportion of nonadditive variancein phenotypic variance are used to perform GS. In addition, taking information on population structure into account can slightly improve prediction performance when the genetic relationship between the training and testing sets is influenced by population stratification due to different allele frequencies. In conclusion, GS is a useful approach for prescreening candidate lines, and the empirical evidence provided by the current study for TRMs and nonadditive effects can inform plant breeding and in turn contribute to the improvement of selection efficiency in practical GS-assisted breeding programs.

Keywords: maize, genomic selection, association and linkage mapping, trait-relevant marker, nonadditive effect, population structure 


\section{INTRODUCTION}

Genomic selection (GS) has been widely implemented to powerfully assist in modern animal and plant breeding $(\mathrm{Xu}$ et al., 2016; Nirea and Meuwissen, 2017; Raoul et al., 2017; Zhang et al., 2017b; Robledo et al., 2018; Brandariz and Bernardo, 2019; Rezende et al., 2019; Sarinelli et al., 2019; Yuan et al., 2019) and has the ability to utilize genome-wide markers (e.g., single nucleotide polymorphisms, SNPs) to accelerate the selection procedure, with the assumption that each marker is associated with minor genetic effects originally proposed by Meuwissen in 2001 in a discussion of several statistical models (Meuwissen et al., 2001). Several factors, including marker density, population size, genetic relationships, statistical models, and breeding platforms, have an impact on the estimation of marker effects that are generally recognized as random effects in the models and thus can impact prediction accuracy (Crossa et al., 2017; Hickey et al., 2017; Schopp et al., 2017; Zhang et al., 2017a; Liu et al., 2018; Wang et al., 2018; Zhang et al., 2019). In general, the estimated effects of each marker follow a normal distribution with the same or different variances under a priori assumptions in a model based on penalized (e.g., ridge regression best linear unbiased prediction, RR-BLUP; genomic BLUP, GBLUP) (Whittaker et al., 2000; VanRaden, 2008; Endelman, 2011) or Bayesian approaches (e.g., BayesA, BayesB, and BayesC) (Meuwissen et al., 2001; Habier et al., 2011). However, some polymorphic markers should be virtually evaluated as having stronger genetic effects and other markers are estimated to have weaker genetic effects because these markers do not have biological functions for the target agronomic traits (Bernardo, 2014; Arruda et al., 2016; Boeven et al., 2016; Spindel et al., 2016; Bian and Holland, 2017). In fact, conventional approaches in quantitative genetics, such as genome-wide association studies (GWASs) and quantitative trait locus (QTL) mapping, can efficiently dissect the genetic architecture of target traits and aid in the exploration of candidate genes for the development of functional markers (Li et al., 2013; Wang et al., 2016; Xiao et al., 2016; Pan et al., 2017; Zhang et al., 2017c). In addition, these functional or trait-relevant markers (TRMs) can explain a large fraction of the genetic variance, which may improve the predictive ability of GS models in plant and animal breeding (Su et al., 2014; Zhang et al., 2014; Ober et al., 2015; Zhang et al., 2015; Arruda et al., 2016; Boeven et al., 2016; Bian and Holland, 2017; Kemper et al., 2018). Several previous studies discussed the advantages of combining GWASs and GS, which usually take TRMs as fixed effects in statistical models (Spindel et al., 2016; Herter et al., 2019; Rice and Lipka, 2019).

With the improvement of statistical models, predication accuracy has increased due to the consideration of supplementary effects, such as nonadditive, fixed, and genotype-by-environment interaction effects (Lopez-Cruz et al., 2015; Zhao et al., 2015; Boeven et al., 2016; Lado et al., 2016; Alves et al., 2019; Herter et al., 2019). Integrating nonadditive effects into statistical models can significantly improve prediction accuracy when the nonadditive variance possesses a relatively large proportion of genetic variance (Su et al., 2012; Azevedo et al., 2015; Liu et al.,
2018; Varona et al., 2018). Furthermore, models including nonadditive effects have been widely applied to evaluate genomic estimated breeding values (GEBVs) of individuals in the process of hybrid selection (Xu et al., 2014; Miedaner et al., 2017; Fristche-Neto et al., 2018; Werner et al., 2018). Compared to a model that considers only additive effects, the improved model can explain more fraction of genetic variance, which can further explore and dissect genetic effects of genomic markers (Da et al., 2014; Bouvet et al., 2016; Morais Júnior et al., 2017; Alves et al., 2019). On the other hand, several previous studies evaluated the effects of population structure with an experimental design in which the genetic distance changed from lessrelevant to morerelevant between training and testing sets (Guo et al., 2014; Isidro et al., 2015; Zhang et al., 2017a; Rio et al., 2019). In fact, population structure that is mainly attributed to different allele frequencies between groups can further impact the construction of genomic relationships and estimation of GEBVs and then affect the predictive ability of GS models (Liu et al., 2018). Information on population structure can be explicitly considered as fixed effects in the models, but significant enhancement of prediction accuracy does not occur (Rio et al., 2019). Furthermore, population structure has less effects on prediction performance when GS is performed with only a specific group or within a subpopulation (Guo et al., 2014). For the description of population structure, principal component analysis (PCA) is an efficient approach based on genomic information (Price et al., 2006). Generally, a PC matrix is used to explicitly illustrate population stratification in GWASs (Price et al., 2006; Huang et al., 2010; Slavov et al., 2014; Huang et al., 2015; Chang et al., 2018) but is rarely applied to correct the effect of population structure in GS models. Hence, taking information on population structure into account will have the benefit of adjusting the bias of estimated marker effects generated by population stratification, likely making great progress in improving prediction performance.

With respect to the application of TRMs in GS, many studies have argued in favor of including TRMs as fixed effects in statistical models to enhance prediction accuracy. However, few reports directly integrating TRMs into GS models have been published (Ober et al., 2015; Yuan et al., 2019). In this study, we primarily aimed to discuss the effect of TRMs and the integration of TRMs and other effects in order to provide recommendations that can assist plant breeders in the design of GS-assisted breeding programs. Phenotypic and genotypic data from three experimental populations, including one natural and two biparental populations, which included six agronomic traits and a $55 \mathrm{~K} \mathrm{SNP}$ array, were collected. Our objectives were to (1) assess the accuracy and quality of association and linkage mapping, (2) evaluate the effect of TRMs identified by association and linkage mapping performed using data from training sets, (3) investigate the degree to which nonadditive effects in combination with TRMs influence prediction accuracy, and (4) integrate information on population structure into a mixed model as a fixed effect to improve predictive ability. Finally, these results were used to provide pertinent advice for improving GS schemes in commercial breeding programs. 


\section{MATERIALS AND METHODS}

\section{Plant Materials and Experimental Management}

The plant materials were described in detail by Liu et al. (2018). In total, three experimental populations, which included one natural and two biparental populations, were used in this study. More specifically, a total of 435 elite maize inbred lines were used to construct the natural population, and the two biparental populations, which were derived from one single-cross maize hybrid with the elite inbred lines Zheng58 and HD568 as parents that included in the natural population, consisted of 212 recombinant inbred lines (RILs) and $304 \mathrm{~F}_{2: 3}$ families, respectively. The natural population was grown in Henan Province in 2014 and 2015. The two biparental populations were evaluated in the same location in 2015 and 2016. A field trial with a randomized incomplete block design was performed with two replicates. Six yield-related agronomic traits constituted the phenotypic data: plant height $(\mathrm{PH}, \mathrm{cm})$, ear height $(\mathrm{EH}, \mathrm{cm})$, ear length $(\mathrm{EL}, \mathrm{mm})$, ear diameter $(\mathrm{ED}, \mathrm{mm})$, grain yield per plant (GYP, kg), and hundred-kernel weight (HKW, g). Furthermore, phenotypic values of HKW and GYP were adjusted to $140 \mathrm{~g} / \mathrm{kg}$ grain moisture.

\section{Statistical Analysis of Phenotypic Data}

Thebest linear unbiased estimates (BLUEs) of genetic effects were estimated using the R package lme4 version 1.1-21 with the following mixed linear model (MLM) (Yang et al., 2017):

$$
y_{i j l}=\mu+g_{i}+e_{l}+g e_{i l}+r_{j l}+\varepsilon_{i j l}
$$

where $y_{i j l}$ is the phenotypic value of the $i^{\text {th }}$ genotype evaluated in the $l^{\text {th }}$ environment with the $j^{\text {th }}$ replicate, $\mu$ is the overall mean, $g_{i}$ is the fixed genetic effect of the $i^{\text {th }}$ individual, $e_{l}$ is the fixed effect of the $l^{\text {th }}$ environment, $g e_{i l}$ is the random interaction effect between the $i^{\text {th }}$ individual and the $l^{\text {th }}$ environment, $r_{j l}$ is the random effect of the $j^{\text {th }}$ replicate within the $l^{\text {th }}$ environment, and $\varepsilon_{i j l}$ is the model residuals. The BLUE values of individuals in each experimental population were used as phenotypic data to perform the subsequent analyses, including a GWAS, QTL mapping, and GS.

\section{Genotyping and Data Analysis}

All inbred lines from the natural and biparental populations were used for genotyping, which was performed with the novel developed maize $55 \mathrm{~K} \mathrm{SNP}$ array (Xu et al., 2017a). As for $\mathrm{F}_{2: 3}$ population, the DNA extracted from leaves of $\mathrm{F}_{2}$ plants was assayed for obtaining genotypic data. Markers with a proportion of missing values $>0.10$ were removed from the three experimental populations. Finally, a total of 38,299 SNPs with minor allele frequencies (MAFs) $>0.05$ were used for further analysis of the natural population. A total of 14,544 and 10,444 SNPs were retained based on chi-square tests $(P>0.01)$ for the RIL and $F_{2: 3}$ populations, respectively. The aim of chi-square test is to screen out markers without segregation distortion in biparental populations.

\section{Genome-Wide Association Study}

Marker-trait association mapping was implemented in the $\mathrm{R}$ package GAPIT version 3.0 with an MLM procedure considering population structure and relative kinship $(\mathrm{Q}+\mathrm{K}$ model)(Price et al., 2006; Yu et al., 2006; Lipka et al., 2012). PCA was conducted with the GAPIT.PCA function in the $\mathrm{R}$ package GAPIT. The determination of PC number was based on a scree test (Cattell, 1966), and the first seven PCs were selected to construct a covariance matrix to avoid the effect of population structure. A significance threshold of $-\log _{10}(P)>4$ for each trait was employed to identify significant association signals for determining the accuracy and quality of association mapping. The R package CMplot version 3.3.3 was used to draw a Manhattan plot (https://github.com/YinLiLin/R-CMplot). The description of candidate genes based on association mapping was based on the maize genetics and genomics database (MaizeGDB, https://www.maizegdb.org/).

\section{Bin Map Construction and QTL Analysis}

The bin maps of the RIL and $\mathrm{F}_{2: 3}$ populations were aligned and constructed with the sliding-window approach to investigate variant calling errors and calculate the ratio of SNP alleles derived from Zheng58 and HD568. A criterion with a window size of 15 adjacent SNPs and a step size of one SNP was applied to scan the genotypic data. Windows with 11 or more continuous SNPs derived from either parent were regarded as homozygous, and those with fewer SNPs from one parent were recognized as heterozygous. Adjacent windows with the same genotype were combined into one block, and these blocks with different genotypes were inferred to be at or near a recombination breakpoint, allowing bin markers to be designated when consecutive blocks lacked a recombination event across all RILs or $\mathrm{F}_{2: 3}$ families (Huang et al., 2009; Zhou et al., 2016). A linkage map was constructed using the Kosambi mapping function and the mstmap function in the $\mathrm{R}$ package ASMap version 1.0-4 (Taylor and Butler, 2017). Identification of QTLs for yield-related agronomic traits was performed by the cim function in the $\mathrm{R}$ package $\mathrm{R} / \mathrm{qtl}$ version $1.44-9$ with composite interval mapping (Arends et al., 2010). Then, 1,000 permutation tests with a significance level of $P<0.05$ were used to determine the threshold likelihood of odds (LOD) ratio for evaluating the significance of each QTL-trait association and for assessing the accuracy and quality of linkage mapping. A 1.5-LOD decrease corresponding to the peak value of the LOD for each bin was defined as the confidence interval for each QTL. Candidate genes identified by linkage mapping were described according to MaizeGDB. In addition, the bin markers were eventually used to perform further GS analysis.

\section{Genomic Selection}

The TRMs detected by association and linkage mapping were used to perform GS for each agronomic trait, and a fivefold cross-validation scheme with 100 replicates was implemented to partition the dataset of each experimental population into training and testing sets and then to calculate the mean correlation coefficient between GEBVs and BLUE values, which 
represented the prediction accuracy $\left(r_{\mathrm{MG}}\right)$. Furthermore, the number of selected markers, including TRMs and randomly selected markers, was set using seven to eight levels (i.e., 20, $50,100,500,1,000,5,000,10,000,30,000$, and all markers in the natural population; $20,50,100,500,1,000,1,500,2,000$, and all bin markers in the biparental population) to test for a difference in prediction accuracy. As for the TRMs and randomly selected markers, the former was selected according to rank of $-\log _{10}(P$ value) in association mapping or LOD scores in linkage mapping, the latter was stochastically sampled from whole genome.

\section{TRM-Based GBLUP Model: Association and Linkage Mapping for the Training Set}

Each experimental population was initially partitioned into training and testing sets based on the scheme of fivefold crossvalidation, and the training set was used to perform a GWAS or QTL mapping in each cross-validation to identify the TRMs. Subsequently, the GEBVs of individuals in the testing set were estimated by the GBLUP model with different numbers of TRMs, which were compared to the $r_{\mathrm{MG}}$ based on randomly selected markers to assess prediction accuracy. The GBLUP model was fitted using the $\mathrm{R}$ package $B G L R$ version 1.0.8 (Pérez and de los Campos, 2014). The hyperparameter settings were based on the default choices in $\mathrm{R}$ package, andGibbs sampler was run for 10,000 iterations with the first 5,000 samples discarded as burn in. The general GBLUP model can be described as follows:

$$
\boldsymbol{y}=\mathbf{1}_{n} \mu+\boldsymbol{u}+\varepsilon
$$

where $y$ is the vector of phenotypic data, $\mathbf{1}_{n}$ is the $\mathrm{n}$-dimensional vector of ones, $\mu$ is the overall mean, $\boldsymbol{u}$ is the random effects sampled from the normal distribution $N\left(0, \boldsymbol{G} \sigma_{\mathrm{u}}^{2}\right), \boldsymbol{G}$ is the genomic relationship matrix, and $\varepsilon$ is the $\mathrm{n}$-dimensional vector of independent random residuals with the normal distribution $N\left(0, \boldsymbol{I} \sigma_{\varepsilon}^{2}\right)$, in which $\boldsymbol{I}$ is an identity matrix. The $\boldsymbol{G}$ matrix was calculated as follows: let $\boldsymbol{Z}=\left\{z_{i j}\right\}$ be the $n \times m$ matrix of markers, where $n$ is the number of individuals in each population, $m$ is the number of markers, $z_{i j}=0,1$, or 2 for the $j^{\text {th }}$ locus in the $i^{\text {th }}$ individual, and $p_{j}$ is the allele frequency of the $j^{\text {th }}$ marker. The $\boldsymbol{G}$ matrix is $\boldsymbol{W} \boldsymbol{W}^{\prime} / 2 \sum_{\mathrm{k}=1}^{\mathrm{m}} p_{k}\left(1-p_{k}\right)$, where $\boldsymbol{W}=\left(w_{i j}\right)$ with $w_{i j}=z_{i j}$ $2 p_{j}$ (VanRaden, 2008).

\section{Extended GBLUP Model Including Additive, Dominance, and Epistatic Effects} The TRMs (mentioned above) were used to fit GS models, and the extended GBLUP model can be described as (Zhao et al., 2015):

$$
y=\mathbf{1}_{n} \mu+\boldsymbol{u}_{\mathrm{a}}+\boldsymbol{u}_{\mathrm{d}}+\boldsymbol{u}_{\mathrm{aa}}+\varepsilon
$$

where $\boldsymbol{u}_{\mathrm{a}}, \boldsymbol{u}_{\mathrm{d}}$, and $\boldsymbol{u}_{\mathrm{aa}}$ are the vectors of random effects for additive genetic effects (A), dominance effects (D), and additive-byadditive interaction (AA) effects and are assumed to obey the normal distributions $N\left(0, \boldsymbol{G}_{\mathrm{a}} \boldsymbol{\sigma}_{\mathrm{a}}^{2}\right), N\left(0, \boldsymbol{G}_{\mathrm{d}} \boldsymbol{\sigma}_{\mathrm{d}}^{2}\right)$, and $N\left(0, \boldsymbol{G}_{\mathrm{aa}} \boldsymbol{\sigma}_{\mathrm{aa}}^{2}\right)$, respectively, where $\boldsymbol{G}_{\mathrm{a}}, \boldsymbol{G}_{\mathrm{d}}$, and $\boldsymbol{G}_{\mathrm{aa}}$ are the genomic relationship matrixes corresponding to additive, dominance, and epistatic genotypic values, respectively. The form of $\boldsymbol{G}_{\mathrm{a}}$ is identical to that in the general GBLUP model. In addition, the $n \times m$ dominance design matrix $\boldsymbol{D}$ is defined as follows (Pérez and de los Campos, 2014; Zhao et al., 2015):

$$
D=\left\{d_{i j}\right\}=\left\{\begin{array}{l}
-\frac{2 p_{12} p_{22}}{\theta}, \text { if } x_{i j}=0 \\
\frac{4 p_{11} p_{22}}{\theta}, \text { if } x_{i j}=1 \\
-\frac{2 p_{11} p_{12}}{\theta}, \text { if } x_{i j}=2
\end{array}\right.
$$

where $p_{11}$ is the allele frequency of $x_{i j}=0$ at the $j^{\text {th }}$ locus, $p_{12}$ is the allele frequency of $x_{i j}=1$ at the $j^{\text {th }}$ locus, $p_{22}$ is the allele frequency of $x_{i j}=2$ at the $j^{\text {th }}$ locus, and $\theta=p_{11}+p_{22}-\left(p_{11}-p_{22}\right)^{2}$. Thus, the dominance relationship matrix is $G_{\mathbf{d}}=n D^{\prime} D^{\prime} / \operatorname{trace}\left(D D^{\prime}\right)$, where the trace is the sum of all diagonal elements. Then, the epistatic relationship matrix can be calculated by $\boldsymbol{G}_{\mathrm{aa}}=\boldsymbol{G}_{\mathrm{a}} \# \boldsymbol{G}_{\mathrm{a}}$, where the octothorpe denotes the Hadamard product of matrixes. The extended GBLUP models were implemented only for $\mathrm{F}_{2: 3}$ population. Four models were used to perform GS, namely, the $\mathrm{A}, \mathrm{A}+\mathrm{AA}, \mathrm{A}+\mathrm{D}$, and $\mathrm{A}+\mathrm{D}+\mathrm{AA}$ models, and the A model was equivalent to the general GBLUP model. These extended GBLUP models were fitted using the $\mathrm{R}$ package $B G L R$ version 1.0.8 (Pérez and de los Campos, 2014).

\section{Fixed-Effects Model Containing the Principal Component Matrix}

The TRMs (mentioned above) were used to fit the GS models. A PC matrix was constructed by the GAPIT.PCA function in GAPIT with TRMs (Lipka et al., 2012) and then added to the BayesC and GBLUP models as a fixed effect. These fixed-effects models were implemented only for natural population. Hence, these models can be described as follows:

$\boldsymbol{y}=\boldsymbol{X} \boldsymbol{\beta}+\boldsymbol{Z} \boldsymbol{u}+\varepsilon$ for the BayesC + fixed effects model $(\mathrm{BC}+\mathrm{PC})$

$\boldsymbol{y}=\boldsymbol{X} \boldsymbol{\beta}+\boldsymbol{u}+\boldsymbol{\varepsilon}$ for the GBLUP + fixed effects model $(\mathrm{G}+\mathrm{PC})$

where $\boldsymbol{X}$ is the design matrix for fixed effects consisting of PCs, $\beta$ is the vector of fixed effect estimates, $Z$ is the design matrix for random effects in the $\mathrm{BC}+\mathrm{PC}$ model, and $\boldsymbol{u}$ is the vector of random effects in both models. Finally, the prediction accuracy assessed by fixed-effect models was compared with the $r_{\mathrm{MG}}$ estimated by the general GBLUP model with TRMs. The fixedeffects models were fitted using the $\mathrm{R}$ package $B G L R$ version 1.0.8 (Pérez and de los Campos, 2014).

\section{RESULTS}

\section{The Quality and Accuracy of Association and Linkage Mapping}

The BLUE values of individuals in each experimental population were used to perform association and linkage mapping, and frequency distribution diagrams were drawn (Supplementary 
Figure 1, 2, and 3). A total of 11 associated SNPs were identified in the natural population by a GWAS using filtered genotype and phenotypic data, and the number of significant SNPs (i.e., those for which the $P$ value surpassed the threshold) for each agronomic trait ranged from 1 to 4 (Supplementary Figure 4). For linkage mapping, there were 2,450 and 2,826 recombination bins with an average length of 840 and $727 \mathrm{~kb}$ for the RIL and $\mathrm{F}_{2: 3}$ populations, respectively. Moreover, 79.5 and $86.2 \%$ of these bins were $<1.0$ $\mathrm{Mb}$ in segment length for each biparental population. Two highdensity genetic maps were constructed using recombination bins as markers based on a chi-square test. The entire genetic distance of each linkage map was 1,811.3 and 1,205.4 cM, and the average and greatest distances between adjacent markers in the respective biparental population were 0.7 and $4.5 \mathrm{cM}$ for the RIL population and 0.4 and $7.8 \mathrm{cM}$ for the $\mathrm{F}_{2: 3}$ population, respectively (Table $\mathbf{1}$, Supplementary Figure 5). With 1,000 permutation tests, the LOD score thresholds for each agronomic trait were ascertained to indicate the presence of a QTL in a particular genomic region (Supplementary Table 1). In the RIL population, a total of 16 QTL were identified for six agronomic traits, and the amount of phenotypic variation explained by each QTL ranged from 3.21 to $12.77 \%$. However, a total of eight QTL with negative genetic effects decreased the phenotypic values of agronomic traits when the alleles were identical to the parent conferred a low phenotypic value. In the $\mathrm{F}_{2: 3}$ population, $38 \mathrm{QTL}$ were detected for the yield-related traits, and each QTL with a LOD value from 5.17 to 26.7 explained 2.49$21.75 \%$ of the phenotypic variation. Furthermore, in addition to estimating the additive genetic effects in the $\mathrm{F}_{2: 3}$ population by QTL mapping, the dominance effects were derived depending on the heterozygous genotypes (Supplementary Table 2). In addition, a total of five pleiotropic QTL (pQTL) were detected by integrating the information for 53 QTL obtained from the RIL and $\mathrm{F}_{2: 3}$ populations, which were distributed on chromosomes 1, 3, 4, 8, and 9 (Table 2).

\section{Effects of Trait-Relevant Markers on Genomic Selection}

As for TRM-based GS, the TRMs were initially identified by association and linkage mapping using phenotypic and genotypic data of training set, and the $r_{\mathrm{MG}}$ based on TRMs was improved compared with that obtained using stochastic markers to perform cross-validation(Figure 1). In particular, $r_{\mathrm{MG}}$ showed significant enhancement when the number of markers was $<5,000$ in the natural population and 500 in the biparental populations. The degree of improvement in $r_{\mathrm{MG}}$ estimated by TRMs compared to randomly selected markers for the agronomic traits with low broadsense heritability was not greater than that for the traits with high broad-sense heritability (the result of broad-sense heritability for each agronomic trait was based on Liu et al., 2018). For instance, the $r_{\mathrm{MG}}$ based on 20 markers in the RIL population increased from 0.395 to 0.526 for $\mathrm{PH}$ and from 0.137 to 0.242 for GYP. In addition, the $r_{\mathrm{MG}}$ obtained by the TRM-based GBLUP model was the same as that obtained by stochastic markers when the number of markers reached 10,000 for the natural population and 1,000 for the biparental populations (Figure 1). In addition, parallel results were obtained for other agronomic traits, including EH, EL, ED, and HKW, when using empirical data to perform cross-validation by TRM-based GBLUP model (Supplementary Figures 6-8).

TABLE 1 | Summary of the high-density genetic map derived from the RIL and $F_{2: 3}$ populations.

\begin{tabular}{|c|c|c|c|c|c|c|}
\hline Pop. ${ }^{a}$ & Chr. & No. of bins ${ }^{c}$ & $\begin{array}{l}\text { Physical length of map } \\
\text { (Mb) }\end{array}$ & Map length (cM) & $\begin{array}{l}\text { Average genetic } \\
\text { length }(\mathrm{cM})\end{array}$ & $\begin{array}{l}\text { Maximal genetic } \\
\text { length (cM) }\end{array}$ \\
\hline \multirow[t]{11}{*}{ RIL } & 1 & 436 & 301.0 & 309.9 & 0.7 & 3.6 \\
\hline & 2 & 301 & 237.0 & 240.6 & 0.8 & 3.0 \\
\hline & 3 & 292 & 232.1 & 224.4 & 0.8 & 3.4 \\
\hline & 4 & 233 & 246.9 & 156.8 & 0.7 & 4.5 \\
\hline & 5 & 298 & 217.6 & 276.6 & 0.9 & 3.1 \\
\hline & 6 & 202 & 169.2 & 128.3 & 0.6 & 3.8 \\
\hline & 7 & 204 & 176.1 & 141.0 & 0.7 & 3.5 \\
\hline & 8 & 209 & 175.5 & 150.7 & 0.7 & 3.0 \\
\hline & 9 & 132 & 156.0 & 93.2 & 0.7 & 3.1 \\
\hline & 10 & 143 & 149.9 & 89.6 & 0.6 & 4.5 \\
\hline & Total & 2,450 & $2,061.3$ & $1,811.3$ & 0.7 & 4.5 \\
\hline \multirow[t]{11}{*}{$\mathrm{F}_{2: 3}$} & 1 & 507 & 301.0 & 169.3 & 0.3 & 1.7 \\
\hline & 2 & 296 & 237.0 & 127.7 & 0.4 & 3.9 \\
\hline & 3 & 279 & 232.1 & 128.1 & 0.5 & 3.6 \\
\hline & 4 & 271 & 246.9 & 130.8 & 0.5 & 7.8 \\
\hline & 5 & 336 & 217.6 & 125.3 & 0.4 & 2.9 \\
\hline & 6 & 308 & 169.2 & 110.8 & 0.4 & 2.2 \\
\hline & 7 & 233 & 176.1 & 95.4 & 0.4 & 3.0 \\
\hline & 8 & 231 & 175.5 & 110.2 & 0.5 & 2.3 \\
\hline & 9 & 181 & 156.0 & 111.6 & 0.6 & 7.1 \\
\hline & 10 & 184 & 149.9 & 96.0 & 0.5 & 2.8 \\
\hline & Total & 2,826 & $2,061.3$ & $1,205.4$ & 0.4 & 7.8 \\
\hline
\end{tabular}

aPop.: the experimental populations.

${ }^{b}$ Chr.: the number of chromosomes.

cNo. of bins: the number of bin markers on the chromosome. 
TABLE 2 | Pleiotropic QTL (pQTL) for each agronomic trait in the biparental populations.

\begin{tabular}{|c|c|c|c|c|c|}
\hline pQTLa & Chr.b & Intervalc (Mb) & Physical length ${ }^{d}(\mathrm{Mb})$ & No. of QTL & Integrated QTL \\
\hline pQTL1 & 1 & $213.1-251.7$ & 38.6 & 4 & $\begin{array}{c}\text { qReh1-2, } q \text { Rhkw1, qFph1-2, } \\
\text { qFhkw1 }\end{array}$ \\
\hline pQTL3 & 3 & 212.0-216.2 & 4.2 & 2 & qFph3, qFeh3 \\
\hline pQTL4 & 4 & $33.7-130.7$ & 97.0 & 2 & qRel4, qFel4 \\
\hline pQTL8 & 8 & $165.2-168.7$ & 3.5 & 2 & qRph8, qReh8 \\
\hline pQTL9 & 9 & 17.5-93.3 & 57.8 & 6 & $\begin{array}{c}\text { qRed9, qRgyp9, qRhkw9, qFph9, } \\
\text { qFgyp9, qFhkw9 }\end{array}$ \\
\hline
\end{tabular}

aThe name of the pleiotropic QTL includes the information of the number of chromosomes.

${ }^{b}$ Chr.: the number of chromosomes.

c Interval: the confidence interval between two bin markers.

a Physical length: the physical distance between two bin markers based on the B73 genome.

\section{Effects of Nonadditive Effects in the Extended GBLUP Model}

To evaluate the influence of nonadditive effects on $r_{\mathrm{MG}}$, a total of four statistical models were used to perform fivefold crossvalidation in the $F_{2: 3}$ population: the $A, A+A A, A+D$, and $\mathrm{A}+\mathrm{D}+\mathrm{AA}$ models. There two agronomic traits, including $\mathrm{PH}$ with high broad-sense heritability and GYP with low broadsense heritability, were selected to elucidate and demonstrate the utility of including multiple effects in GS models (the broad-sense heritabilityestimated by Liu et al., 2018, 0.83 for PH and 0.65 for GYP). There was no remarkable improvement when integrating dominance and epistatic effects in the GBLUP model, which a target trait with high broad-sense heritability was used for GS (Figure 2). However, compared to the GBLUP model that considered only additive effects, the GS model with nonadditive effects significantly enhanced the $r_{\mathrm{MG}}$ for the traits with low broad-sense heritability. On the other hand, for GYP, statistical models including additive and dominance effects were clearly superior to the additive and epistatic model, and this superiority was consistent across various situations with diverse marker densities. In addition, a slight improvement in $r_{\mathrm{MG}}$ was observed between the $\mathrm{A}+\mathrm{D}+\mathrm{AA}$ and $\mathrm{A}+\mathrm{D}$ models in each situation except that in which 20 TRMs were used to fit extended GBLUP models, but the $r_{\text {MG }}$ was highest for the GBLUP model considering additive, dominance, and epistatic effects. Furthermore, the improvement in $r_{\mathrm{MG}}$ between the A + D and $\mathrm{A}+\mathrm{D}+\mathrm{AA}$ models became increasingly large as more TRMs were used to construct the genomic relationship matrix. For example, the enhancement of $r_{\mathrm{MG}}$ increased from 0.003 to 0.010 as the number of TRMs increased from 50 to 2,000 (Figure 2). In addition, the proportion of genetic variance explained by dominance effects in the $\mathrm{A}+\mathrm{D}$ and $\mathrm{A}+\mathrm{D}+\mathrm{AA}$ models was 0.205 and 0.173 , respectively. However, the proportion of genetic variance explained by AA interaction effects was 0.127 and 0.032 for the $\mathrm{A}+$ AA and A + D + AA models, respectively (Table 3).

\section{Using the Information of Population Structure as Fixed Effects to Improve the Predictive Ability of GS Models}

The possibility of enhancing $r_{\mathrm{MG}}$ through improved models was investigated using TRMs to construct PC matrix that was appointed as fixed effects and incorporated in models. When $\mathrm{PH}$ was used for cross-validation in the case where more than 10,000 TRMs were applied in GS, the $r_{\mathrm{MG}}$ was slight improvement when PC matrix was included as a fixed effect in the BayesC and GBLUP models (Figure 3A). However, for GYP, which had low broad-sense heritability, including population structure information as a fixed effect in GS models enhanced their predictive ability at a moderate marker density. For instance, the $r_{\mathrm{MG}}$ estimated by $100 \mathrm{TRMs}$ was 0.248 for the general GBLUP model, 0.260 for the BC + PC model, and 0.262 for the G + PC model. Moreover, the maximum degree of improvement in $r_{\mathrm{MG}}$ was 0.015 when 500 TRMs were used to obtain the PCs for the corresponding design matrix included as a fixed effect in the $\mathrm{BC}+\mathrm{PC}$ model. The predictive ability of the $\mathrm{G}+\mathrm{PC}$ model was higher than that of the BC + PC model in some situations when the number of TRMs was $<1,000$ (Figure $3 \mathbf{B}$ ). However, the superiority of the fixed models to the general GBLUP models with TRMs was small when the number of markers was $>5,000$.

\section{DISCUSSION}

GS, the theoretical and practical application of marker-assisted selection, has been widely implemented in animal and plant molecular breeding with the accumulation of genotypic and phenotypic data in commercial and experimental breeding programs (Nirea and Meuwissen, 2017; Raoul et al., 2017; Xu et al., 2018; Mastrodomenico et al., 2019; Rezende et al., 2019; Sarinelli et al., 2019; Yuan et al., 2019). Several factors, such as population size, population structure, marker density, heritability, statistical models, and genetic relationships between training and breeding populations, affect prediction accuracy (Schopp et al., 2017; Zhang et al., 2017a; Cerrudo et al., 2018; Edwards et al., 2019; Zhang et al., 2019). Previous researches have been performed using TRMs to test the benefit of employing candidate loci with biological functions based on historical or experimental information (Arruda et al., 2016; Boeven et al., 2016; Rice and Lipka, 2019; Yuan et al., 2019). However, TRMs are usually treated as fixed effects in statistical models. In this study, we aimed to evaluate and discuss the effect of TRMs in 


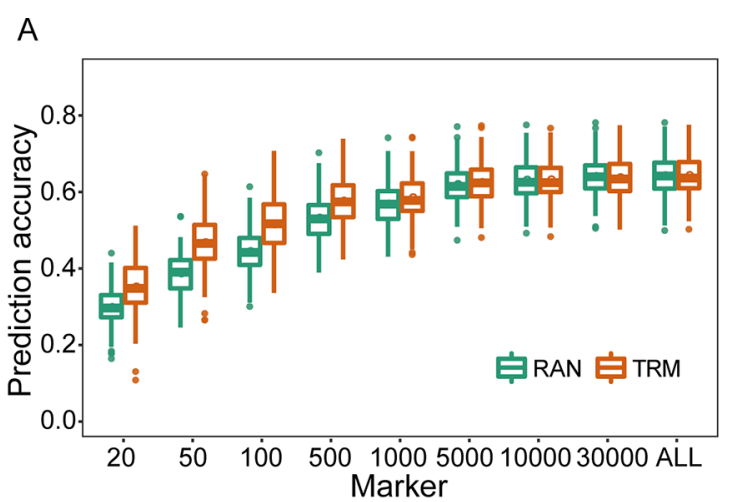

C

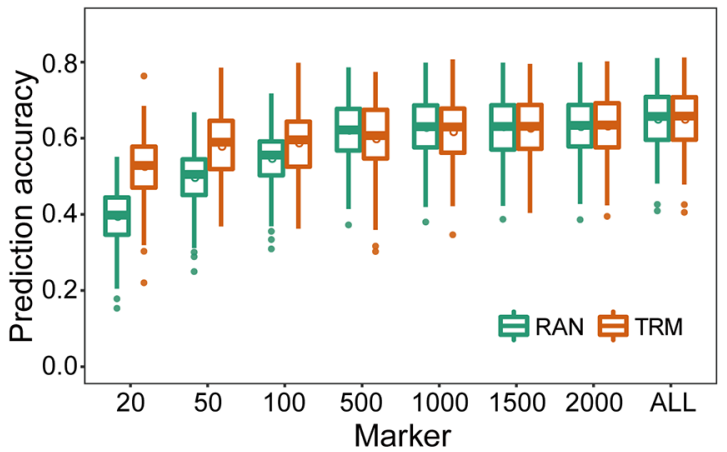

$\mathrm{E}$

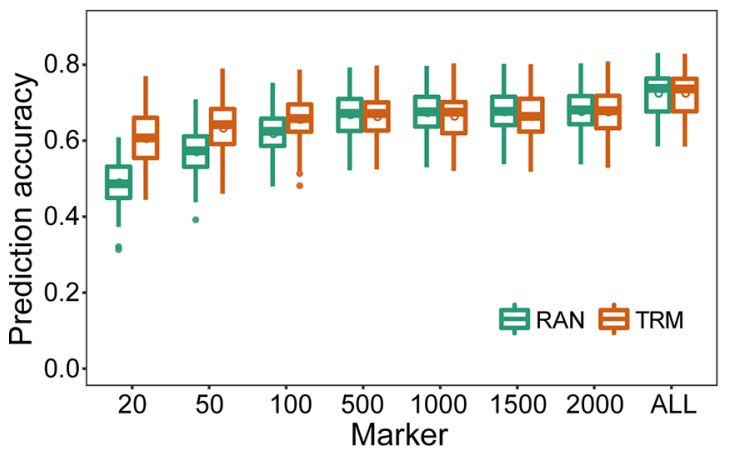

B

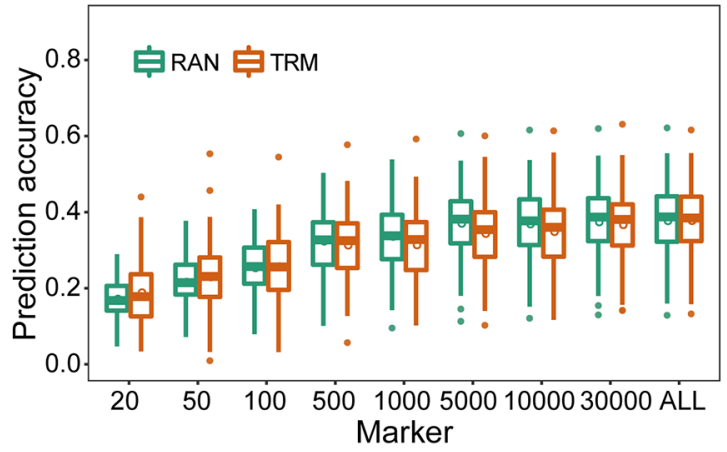

D

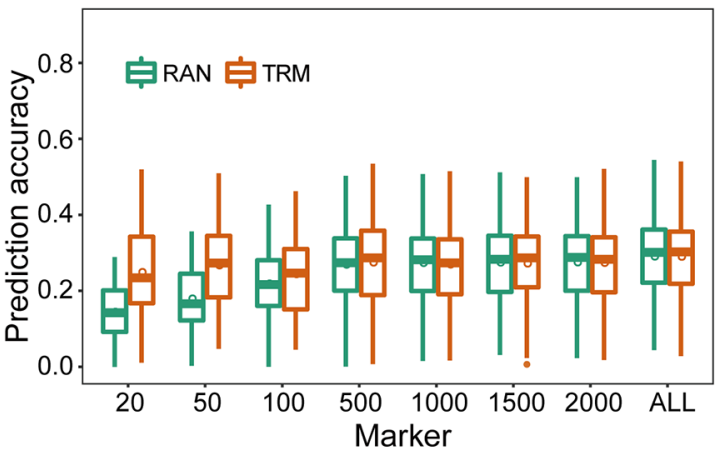

$\mathrm{F}$

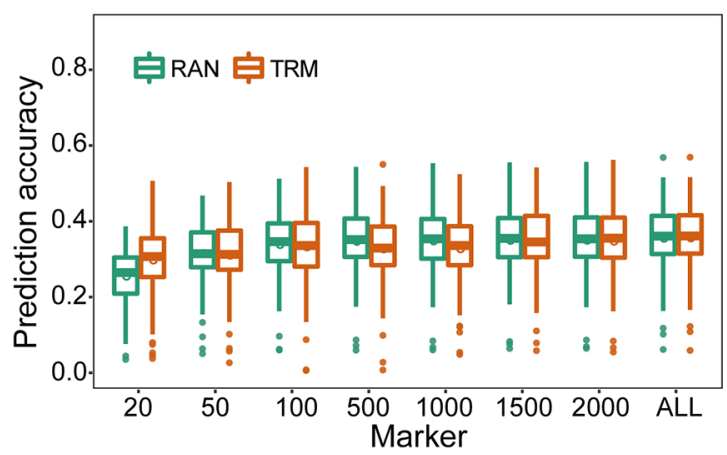

FIGURE 1 | Comparison of prediction accuracies between trait-relevant markers (TRMs) and randomly selected markers based on the results of association and linkage mapping using genotypic and phenotypic data of the training set within the experimental populations. (A) and (B) Plant height (PH) and grain yield per plant (GYP) in the natural population ( $N=435)$; (C) and (D) PH and GYP in the RIL population ( $N=212)$; (E) and (F) PH and GYP in the $F_{2: 3}$ population ( $\left.N=304\right)$. $N$ is the number of individuals in each population. TRM: the prediction accuracy based on TRMs in the general genomic best linear unbiased prediction (GBLUP) model; RAN: the prediction accuracy based on randomly selected markers in the general GBLUP model. ALL: total of 38,299 single nucleotide polymorphisms (SNPs), 2,450 and 2,826 bin markers were used to perform the scheme of cross-validation in natural, recombinant inbred line (RIL), and $\mathrm{F}_{2: 3}$ populations, respectively. The fivefold cross-validation scheme was implemented in this case.

various situations using empirical data and proposed statistical models with the purpose of reducing the effect of population structure on prediction accuracy.

Association and linkage mapping are efficient strategies for dissecting the genetic architecture of target traits, and traitrelevant locican then be used to accelerate the breeding process and assist in improving selection efficiency. According to the results of a GWAS of yield-related traits, two candidate genes related to GYP and HKW were identified by searching the MaizeGDB database, namely, GRMZM2G373928 and GRMZM2G044744, which are located on chromosome 8 (Supplementary Table 3). The candidate genes are ZCN14 and ZmSSIV, the first of which is expressed in the tassel, ear primordium, and endosperm and is involved in the early development of kernels (Danilevskaya et al., 2008). The highest expression of the second candidate was detected in the embryo, endosperm, and pericarp 15 days after pollination, and the specific function of this gene is the regulation of starch granule formation, which further affects crop yield and quality (Liu et al., 2015). In addition, the QTL identified by linkage mapping, including qReh1-2, qRhkw1, 

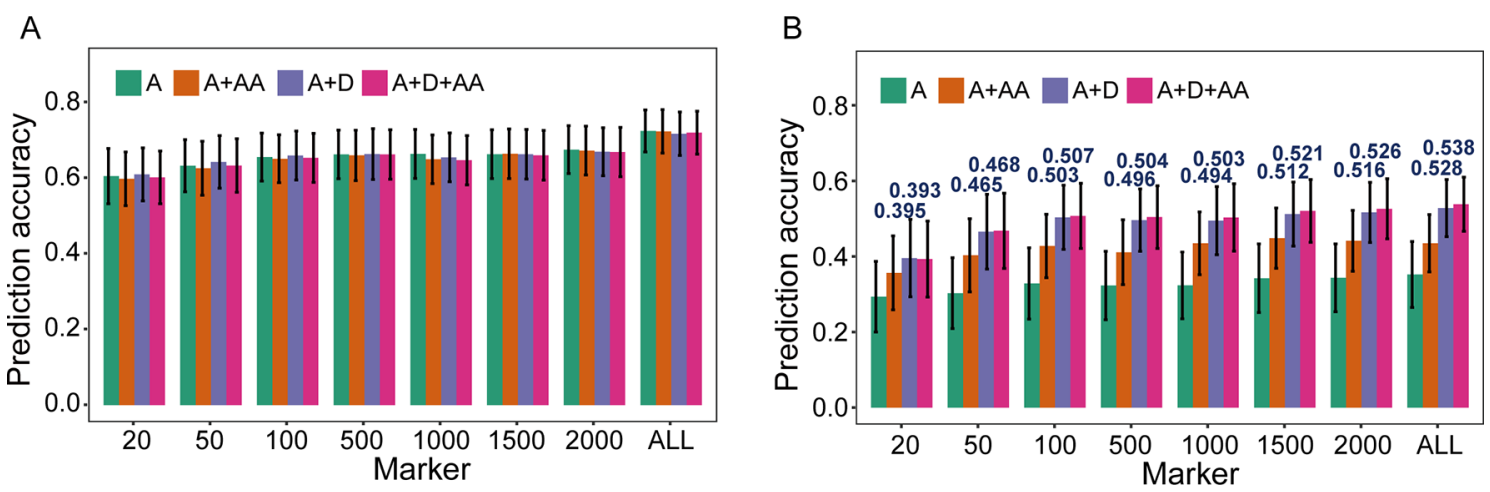

FIGURE 2 | Prediction accuracy of models based on nonadditive effects and trait-relevant markers (TRMs). (A) and (B) Plant height (PH) and grain yield per plant (GYP) in the $F_{2 \cdot 3}$ population $(N=304)$. $N$ is the number of individuals in each population. The capital letters $A$, $D$, and $A A$ refer to additive, dominance, and additiveby-additive interaction effects, respectively. The A model that contains only additive effects is equivalent to the general genomic best linear unbiased prediction (GBLUP) model using trait-relevant markers (TRMs) to perform cross-validation. ALL: total of 2,826 bin markers were used to perform the scheme of crossvalidation in $\mathrm{F}_{2: 3}$ population. The fivefold cross-validation scheme was implemented in this case.

TABLE 3 | Proportions of variance components estimated by the models.

\begin{tabular}{|c|c|c|c|c|c|c|c|c|}
\hline \multirow[t]{2}{*}{ Parameters $^{a}$} & \multicolumn{4}{|c|}{$\mathbf{P H}^{\mathrm{b}}$} & \multicolumn{4}{|c|}{ GYP } \\
\hline & $A^{c}$ & $A+\mathbf{A} A$ & $A+D$ & $A+D+A A$ & A & $A+\mathbf{A} A$ & $A+D$ & $A+D+A A$ \\
\hline$\sigma_{\mathrm{a}}^{2}$ & $\begin{array}{c}0.564 \\
(0.023)\end{array}$ & $\begin{array}{c}0.542 \\
(0.023)\end{array}$ & $\begin{array}{c}0.526 \\
(0.025)\end{array}$ & $\begin{array}{c}0.510 \\
(0.024)\end{array}$ & $\begin{array}{c}0.182 \\
(0.014)\end{array}$ & $\begin{array}{c}0.172 \\
(0.017)\end{array}$ & $\begin{array}{c}0.171 \\
(0.016)\end{array}$ & $\begin{array}{c}0.161 \\
(0.017)\end{array}$ \\
\hline$\sigma_{\mathrm{d}}^{2}$ & & & $\begin{array}{c}0.043 \\
(0.006)\end{array}$ & $\begin{array}{c}0.036 \\
(0.004)\end{array}$ & & & $\begin{array}{c}0.205 \\
(0.022)\end{array}$ & $\begin{array}{c}0.173 \\
(0.022)\end{array}$ \\
\hline$\sigma_{\mathrm{aa}}^{2}$ & & $\begin{array}{c}0.032 \\
(0.005)\end{array}$ & & $\begin{array}{c}0.019 \\
(0.003)\end{array}$ & & $\begin{array}{l}0.127 \\
(0.02)\end{array}$ & & $\begin{array}{c}0.032 \\
(0.007)\end{array}$ \\
\hline$\sigma_{\varepsilon}^{2}$ & $\begin{array}{c}0.436 \\
(0.023)\end{array}$ & $\begin{array}{c}0.425 \\
(0.024)\end{array}$ & $\begin{array}{c}0.431 \\
(0.024)\end{array}$ & $\begin{array}{c}0.435 \\
(0.025)\end{array}$ & $\begin{array}{c}0.818 \\
(0.014)\end{array}$ & $\begin{array}{c}0.700 \\
(0.028)\end{array}$ & $\begin{array}{c}0.624 \\
(0.028)\end{array}$ & $\begin{array}{c}0.634 \\
(0.026)\end{array}$ \\
\hline
\end{tabular}

${ }^{a} \sigma_{\mathrm{a}}^{2}$ : additive genetic variance; $\sigma_{\mathrm{d}}^{2}$ :dominance variance; $\sigma_{\mathrm{aa}}^{2}$ :additive-by-additive interaction variance; $\sigma_{\varepsilon}^{2}$ :estimated error variance.

${ }^{b}$ PH: plant height; GYP: grain yield per plant.

${ }^{c}$ The model containing various effects; $A$, additive genetic effect; $D$, dominance effect; $A A$, additive-by-additive interaction effect. The $B L U E$ values of individuals in $F_{2: 3}$ population were used as phenotypic data. The extended GBLUP models were implemented for each trait in the $F_{2: 3}$ population using 2,000 TRMs. The numbers in the parentheses were standard deviation.
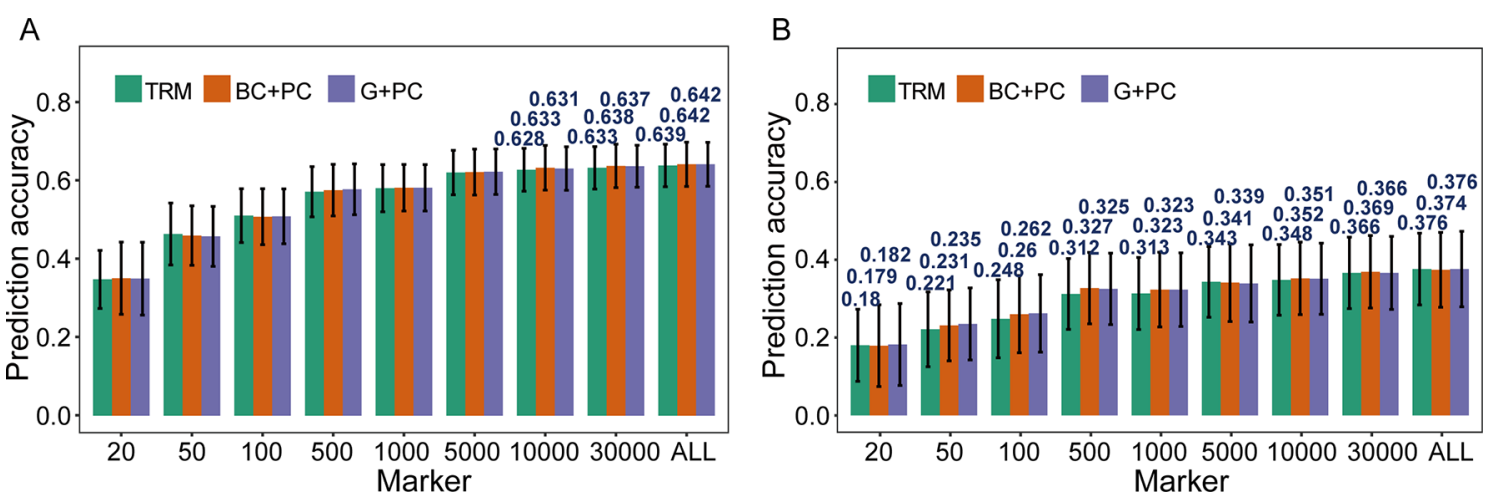

FIGURE 3 | Comparison of prediction accuracy between models based on trait-relevant markers (TRMs). (A) and (B) Plant height (PH) and grain yield per plant (GYP) in the natural population ( $N=435) . N$ is the number of individuals in each population; trait-relevant marker (TRM): the prediction accuracy based on TRMs in the general genomic best linear unbiased prediction (GBLUP) model; BC + PC: the prediction accuracy based on the BayesC model with PCs as fixed effects using TRMs; G + PC: the prediction accuracy based on the GBLUP model with PCs as fixed effects using TRMs. ALL: total of 38,299 SNPs were used to perform the scheme of cross-validation in natural population. The fivefold cross-validation scheme was implemented in this case. 
$q F g y p 5, q F h k w 3-2$, and $q F h k w 7$, are likely important for yieldrelated traits; the IDs of the corresponding candidate genes are GRMZM2G103773, GRMZM2G018627, GRMZM2G121468, GRMZM5G803935, and AC207722.2_FG009, respectively (Supplementary Table 3). In previous studies, these candidate genes were described as having the functions of internode length regulation and photosystem and floral development. The BRD1 gene associated with $\mathrm{EH}$ is essential for internode elongation, and its mutants in maize exhibit severe dwarfism (Makarevitch et al., 2012; Peiffer et al., 2014). The genes Lhcb2 and Lhcb9 can encode an apoprotein of light-harvesting chlorophyll-binding protein, which captures light energy for photosystem II (Viret et al., 1993; Boldt and Scandalios, 1995). The VP15 gene is related to abscisic acid biosynthesis and formation of viviparous seed, and is expressed in both the endosperm and embryo during seed maturation (Suzuki et al., 2006). The gene TS4 encodes a mir172 miRNA, which indirectly regulates spikelet meristem determinacy (Xu et al., 2017b). In addition, four QTL with overlapping intervals were detected in the RIL and $F_{2: 3}$ populations, namely, qReh1-2, qRhkw1, qFph1-2, and $q F h k w 1$, which were linked to QTL affecting PH and yield (Table 2). This important region encompasses at least two genes based on the MaizeGDB database, specifically, BRD1 and Lhcb9, implying that pleiotropy or close linkage to other QTL related to various traits exists in this region and further indicating that the genes can be simultaneously inherited by various generations of segregating populations that were constructed by common parents. Moreover, the region might be a hotspot that consists of important QTL with the biological function of controlling $\mathrm{PH}$ and kernel weight; understanding the genetic basis of these traits will enable plant breeders to achieve the full yield potential of maize.

GS is the process of using phenotypic and genotypic data from training populations to estimate the GEBVs of individuals in breeding populations based on their genotypic values (Jonas and de Koning, 2013; Desta and Ortiz, 2014; Crossa et al., 2017). Genomic relationships, population structure, and genetic distance can be revealed by genotypic data in combination with statistical and genetic approaches, which will have a crucial impact on the prediction accuracy in GS (Guo et al., 2014; Isidro et al., 2015; Rio et al., 2019). Hence, the number and genetic effects of molecular markers are of great importance in achieving a better $r_{\text {MG }}$ (Su et al., 2014; Zhang et al., 2014; Ober et al., 2015; Zhang et al., 2015; Arruda et al., 2016; Boeven et al., 2016; Yuan et al., 2019). As for TRM-based GS in this study, the association and linkage mapping were first performed using phenotypic and genotypic data of individuals within the training set; TRMs derived from the training set were used to estimate $r_{\mathrm{MG}}$. The results in this study illustrate that TRMs can enhance $r_{M G}$ in most situations, especially when the marker density within natural and biparental populations is low, as shown by the use of 20-500 functional markers to perform cross-validation and achieve better prediction performance. In this respect, our results are similar to those of several reports that verified the advantage of applying TRMs in GS (Spindel et al., 2016; Yuan et al., 2019). In addition, for biparental populations with comparatively simple population structure and a lower genetic distance between the training and testing sets, the increase in $r_{\mathrm{MG}}$ is extremely large when a small number of TRMs are used in cross-validation, especially when $r_{\mathrm{MG}}$ is based on 50 TRMs, in which case, it is approximately equal to the maximum obtained when all randomly selected markers are used to predict the GEBVs of individuals in the testing set. This method can greatly reduce the costs of genotyping in GS-assisted breeding. Regarding the natural population with complex population structure and a greater genetic distance between subgroups, the degree of $r_{\mathrm{MG}}$ improvement was extremely small when using a few TRMs compared to that obtained with biparental populations. Thus, population structure and genetic relationships may have negative effects on $r_{\mathrm{MG}}$, and this explanation is supported by previous empirical studies (Guo et al., 2014; Spindel et al., 2015; Rio et al., 2019), which will be further discussed below. On the other hand, it may require extra expenditure when the criteria centered on multiple breeding targets or agronomic traits were used for selection in a breeding program, which the TRMs may vary from one agronomic trait to another. The utilization of overlapped TRMs may be more important and significant in the GS-assisted breeding schemes. In this study, a better prediction performance for different traits were obtained when total of 500-1,000 overlapped TRMs based on the results that all individuals of each population were used to perform association and linkage mapping were applied to implement GS (Supplementary Figure 9). Despite that, the application of overlapped TRMs can be limited because the TRMs were different between traits within various experimental populations and agronomic traits had disparate genetic basis and complexity. Hence, the profound study of molecular mechanism of target traits should be required with the purpose of improving the availability and practicability of TRMs in GS breeding programs. In brief, TRMs can be conducive to improving the predictive ability of models and reducing breeding costs for further enhancing genetic gain.

Nonadditive variance, which includes dominance and epistatic effects that generally consist of various interaction effects, such as AA, additive-by-dominance (AD), and dominance-bydominance (DD) interaction effects, has long been recognized as essential component for dissecting the genetic architecture of target traits and understanding the genetic basis of quantitative traits (Su et al., 2012; Da et al., 2014; Muñoz et al., 2014; Azevedo et al., 2015; Jiang and Reif, 2015; Zhao et al., 2015; Bouvet et al., 2016; Dias et al., 2018; Liu and Chen, 2018; Varona et al., 2018). Several studies were performed using TRMs to test the effect of various combinations of nonadditive effects in extended GBLUP models. For the traits with high heritability, the proportion of nonadditive variance in the phenotypic variance was so small that the degree of $r_{\mathrm{MG}}$ improvement when the linear mixed model included dominance and epistatic effects had almost no change compared with that obtained with the general GBLUP model considering only additive effects (Wang et al., 2017; Dias et al., 2018; Liu et al., 2018; Alves et al., 2019). For example, PH in the $\mathrm{F}_{2: 3}$ population has low proportion of nonadditive variance estimated by extended GBLUP models in this study, and then, there was no significant difference between models accounting for various effects, which validates the argument made above. In other words, additive genetic effects can explain the vast majority of genetic variancewhen genomic prediction is implemented for 
traits with high heritability, which is in agreement with the results of several other studies (Melchinger et al., 2003; Fischer et al., 2008; Hallauer et al., 2010). However, for the traits with high proportion of nonadditive variance, such as GYP in this study, a great improvement in $r_{\mathrm{MG}}$ was achieved when nonadditive effects were included in statistical models. Furthermore, compared to the additive-effects model, the extent of $r_{\mathrm{MG}}$ improvement in the $\mathrm{A}+\mathrm{D}$ model was greater than that in the A + AA model, regardless of how many TRMs were used in cross-validation. This phenomenon may have occurred because considering dominance effects in the predictive model can account for a higher proportion of the genetic variance than including AA interaction effects in the model. Hence, a GS model including additive and dominance effects for a $\mathrm{F}_{2: 3}$ population with genotypes displaying a high ratio of heterozygous alleles can have important effects on $r_{\mathrm{MG}}$, and the influence of dominance effects on the enhancement of $r_{\mathrm{MG}}$ under such conditions can sometimes be superior to that when epistatic effects are considered in GS models. These results were similar to the results of some previous studies (Su et al., 2012; Da et al., 2014; Bouvet et al., 2016; Alves et al., 2019). The largest $r_{\mathrm{MG}}$ was obtained when the $\mathrm{A}+\mathrm{D}+\mathrm{AA}$ model was implemented in cross-validation, illustrating that fully considering nonadditive effects in extended GS models can further improve predictive performance. However, models including more epistatic effects, such as $\mathrm{AA}, \mathrm{AD}$, and $\mathrm{DD}$ interaction effects, exhibited a poorer $r_{M G}$ than the $\mathrm{A}+\mathrm{D}+\mathrm{AA}$ model and even than the A + D model (results not shown). This phenomenon may result because considering more effects in models can increase their complexity and affect the goodness of fit (Alves et al., 2019). On the other hand, it may be attributed to epistatic effects redundantly involving other effects when various matrixes of genomic relationships are constructed to dissect genetic variance (Hallauer et al., 2010). For instance, additive and dominance effects may be repeatedly considered in other interaction effects, thereby potentially having an undesirable impact on the accuracy of marker effect estimates (Plieschke et al., 2015); however, further study is required to fully reveal the reasons for this finding. In general, to achieve better prediction performance in a heterozygous population, nonadditive effects should be taken into account to enhance predictive ability and accelerate the breeding process.

The formation of population structure and genetic relationships can impact the accuracy of estimates of marker effects in stratified populations and further affect the prediction performance in GS (Technow et al., 2013; Guo et al., 2014; Spindel et al., 2015; Duangjit et al., 2016; Habyarimana, 2016; Rio et al., 2019). There are at least two approaches for reducing the influence of population structure on $r_{\mathrm{MG}}$. The first is constructing the training population to be closely related to the breeding population before implementing GS (Toosi et al., 2010; Esfandyari et al., 2015; Zhang et al., 2017a). The second is considering the information of population structure as a fixed effect in the model (Guo et al., 2014; Rio et al., 2019). In the case of the former, we investigated the effects of genetic relationships between training and testing sets in a previous publication (Liu et al., 2018). In this study, the effects of population structure on $r_{\mathrm{MG}}$ were examined using various numbers of TRMs in the models and then discussed.
Previous research has explicitly taken genetic structure into account using all markers to fit the modified models without considering TRMs, and $r_{\text {MG }}$ was not significantly improved compared to that obtained by a general GBLUP model (Rio et al., 2019). The results from this study were similar to those of the abovementioned research when the number of TRMs was $>5000$ for GYP. In addition, there was a slight improvement in $r_{\mathrm{MG}}$ when more than 10,000 TRMs were used to perform GS. Therefore, using information of genetic structure estimated with TRMs as a fixed effect can enhance the $r_{\mathrm{MG}}$ to some extent and may improve the proportion of genetic variance explained by the mixed model. The impact of population structure is attributed to the difference in allele frequencies between groups, which likely affects the estimation of marker effects and cannot be captured by the general parameters of models (Liu et al., 2018; Rio et al., 2019). Hence, developing advanced models that take such information into account will be required to achieve better prediction performance in the future.

\section{CONCLUSIONS}

GS has developed with high-throughput genotyping technology and is a landmark for theoretical exploration, from targeting individual loci to considering the whole genome, in the field of animal and plant molecular breeding. Empirical genotypic and phenotypic data from three experimental populations were used to investigate the effects of including TRMs and nonadditive effects in GS models. We found that the $r_{\mathrm{MG}}$ based on TRMs was better than that obtained by stochastically selected markers, and a few TRMs resulted in a higher $r_{M G}$ in biparental populations with simple population structure. In addition, considering nonadditive effects, including dominance, epistatic, and fixed effects, in the statistical models further improved the predictive ability for accelerating the breeding process in cooperation with TRMs. On the other hand, the utilization of TRMs in GS can ensure a sufficient $r_{\mathrm{MG}}$ for selecting candidate lines and optimize the cost of the breeding cycle, with the strong potential to increase benefits. However, the development of appropriate GS models in the future should take nonadditive effects and information of population structure into account, which can fully capture dominance and epistatic effects on the evaluation of potential hybrids and reduce the effects of population structure, enabling adequate predictive performance when the training and breeding populations are very genetically distant.

\section{DATA AVAILABILITY}

The datasets generated for this study are available on request to the corresponding author.

\section{AUTHOR CONTRIBUTIONS}

$\mathrm{CH}$ and $\mathrm{HW}$ conceived and designed the experiments. XL and HW performed the experiments. XL and HW analyzed the data. $\mathrm{XH}, \mathrm{KL}, \mathrm{ZL}$, and YW contributed materials/analysis tools. XL and $\mathrm{HW}$ wrote the paper. 


\section{FUNDING}

This study was supported by the National Key Research and Development Program of China (Grant No. 2017YFD0101201), the Agricultural Science and Technology Innovation Program at CAAS, and the National Basic Research Program of China (973 Program) (Grant No. 2014CB138200).

\section{ACKNOWLEDGMENTS}

The authors thank the anonymous reviewers for valuable comments and suggestions that improved the manuscript, and Dr. Yunbi $\mathrm{Xu}$,

\section{REFERENCES}

Alves, F. C., Granato, Í.S.C., Galli, G., Lyra, D. H., Fritsche-Neto, R., and de los Campos, G. (2019). Bayesian analysis and prediction of hybrid performance. Plant Methods 15, 14. doi: 10.1186/s13007-019-0388-x

Arends, D., Prins, P., Jansen, R. C., and Broman, K. W. (2010). R/qtl: highthroughput multiple QTL mapping. Bioinformatics 26, 2990-2992. doi: 10.1093/bioinformatics/btq565

Arruda, M. P., Lipka, A. E., Brown, P. J., Krill, A. M., Thurber, C., Brown-Guedira, G., et al. (2016). Comparing genomic selection and marker-assisted selection for Fusarium head blight resistance in wheat (Triticum aestivum L.). Mol. Breed. 36, 84. doi: 10.1007/s11032-016-0508-5

Azevedo, C. F., de Resende, M. D. V., e Silva, F. F., Viana, J. M. S., Valente, M. S. F., Resende, M. F. R., et al. (2015). Ridge, Lasso and Bayesian additive-dominance genomic models. BMC Genet. 16, 105. doi: 10.1186/s12863-015-0264-2

Bernardo, R. (2014). Genomewide selection when major genes are known. Crop Sci. 54, 68-75. doi: 10.2135/cropsci2013.05.0315

Bian, Y., and Holland, J. B. (2017). Enhancing genomic prediction with genomewide association studies in multiparental maize populations. Heredity 118, 585. doi: 10.1038/hdy.2017.4

Boeven, P. H. G., Longin, C. F. H., Leiser, W. L., Kollers, S., Ebmeyer, E., and Würschum, T. (2016). Genetic architecture of male floral traits required for hybrid wheat breeding. Theor. Appl. Genet. 129, 2343-2357. doi: 10.1007/ s00122-016-2771-6

Boldt, R., and Scandalios, J. G. (1995). Circadian regulation of the Cat 3 catalase gene in maize (Zea mays L.): entrainment of the circadian rhythm of Cat 3 by different light treatments. Plant J. 7, 989-999. doi: 10.1046/j.1365-313X.1995.07060989.x

Bouvet, J.-M., Makouanzi, G., Cros, D., and Vigneron, P. (2016). Modeling additive and non-additive effects in a hybrid population using genome-wide genotyping: prediction accuracy implications. Heredity 116, 146-157. doi: 10.1038/hdy.2015.78

Brandariz, S. P., and Bernardo, R. (2019). Small ad hoc versus large general training populations for genomewide selection in maize biparental crosses. Theor. Appl. Genet. 132, 347-353. doi: 10.1007/s00122-018-3222-3

Cattell, R. B. (1966). The scree test for the number of factors. Multivariate Behav. Res. 1, 245-276. doi: 10.1207/s15327906mbr0102_10

Cerrudo, D., Cao, S., Yuan, Y., Martinez, C., Suarez, E. A., Babu, R., et al. (2018). Genomic selection outperforms marker assisted selection for grain yield and physiological traits in a maize doubled haploid population across water treatments. Front. Plant Sci. 9, 366. doi: 10.3389/fpls.2018.00366

Chang, F., Guo, C., Sun, F., Zhang, J., Wang, Z., Kong, J., et al. (2018). Genomewide association studies for dynamic plant height and number of nodes on the main stem in summer sowing soybeans. Front. Plant Sci. 9, 1184. doi: 10.3389/ fpls.2018.01184

Crossa, J., Pérez-Rodríguez, P., Cuevas, J., Montesinos-López, O., Jarquín, D., de los Campos, G., et al. (2017). Genomic selection in plant breeding: methods, models, and perspectives. Trends Plant Sci. 22, 961-975. doi: 10.1016/j. tplants.2017.08.011

Da, Y., Wang, C., Wang, S., and Hu, G. (2014). Mixed model methods for genomic prediction and variance component estimation of additive and dominance
Institute of Crop Sciences, Chinese Academy of Agricultural Sciences (CAAS) for technical assistance. This study was supported by the National Key Research and Development Program of China (Grant No. 2017YFD0101201), the Agricultural Science and Technology Innovation Program at CAAS, and the National Basic Research Program of China (973 Program) (Grant No. 2014CB138200).

\section{SUPPLEMENTARY MATERIAL}

The Supplementary Material for this article can be found online at: https://www.frontiersin.org/articles/10.3389/fpls.2019.01129/ full\#supplementary-material effects using SNP markers. PLoS One 9, e87666. doi: 10.1371/journal. pone.0087666

Danilevskaya, O. N., Meng, X., Hou, Z., Ananiev, E. V., and Simmons, C. R. (2008). A genomic and expression compendium of the expanded PEBP gene family from maize. Plant Physiol. 146, 250-264. doi: 10.1104/pp.107.109538

Desta, Z. A., and Ortiz, R. (2014). Genomic selection: genome-wide prediction in plant improvement. Trends Plant Sci. 19, 592-601. doi: 10.1016/j. tplants.2014.05.006

Dias, K. O. D. G., Gezan, S. A., Guimarães, C. T., Nazarian, A., da Costa e Silva, L., Parentoni, S. N., et al. (2018). Improving accuracies of genomic predictions for drought tolerance in maize by joint modeling of additive and dominance effects in multi-environment trials. Heredity 121, 24-37. doi: 10.1038/ s41437-018-0053-6

Duangjit, J., Causse, M., and Sauvage, C. (2016). Efficiency of genomic selection for tomato fruit quality. Mol. Breed. 36, 29. doi: 10.1007/s11032-016-0453-3

Edwards, S. M., Buntjer, J. B., Jackson, R., Bentley, A. R., Lage, J., Byrne, E., et al. (2019). The effects of training population design on genomic prediction accuracy in wheat. Theor. Appl. Genet. 132, 1943-1952. doi: 10.1007/s00122-019-03327-y

Endelman, J. B. (2011). Ridge regression and other kernels for genomic selection with R package rrBLUP. Plant Genome 4, 250-255. doi: 10.3835/ plantgenome2011.08.0024

Esfandyari, H., Sørensen, A. C., and Bijma, P. (2015). A crossbred reference population can improve the response to genomic selection for crossbred performance. Genet. Sel. Evol. 47, 76. doi: 10.1186/s12711-015-0155-Z

Fischer, S., Möhring, J., Schön, C. C., Piepho, H.-P., Klein, D., Schipprack, W., et al. (2008). Trends in genetic variance components during 30 years of hybrid maize breeding at the University of Hohenheim. Plant Breed. 127, 446-451. doi: 10.1111/j.1439-0523.2007.01475.x

Fristche-Neto, R., Akdemir, D., and Jannink, J.-L. (2018). Accuracy of genomic selection to predict maize single-crosses obtained through different mating designs. Theor. Appl. Genet. 131, 1153-1162. doi: 10.1007/s00122-018-3068-8

Guo, Z., Tucker, D. M., Basten, C. J., Gandhi, H., Ersoz, E., Guo, B., et al. (2014). The impact of population structure on genomic prediction in stratified populations. Theor. Appl. Genet. 127, 749-762. doi: 10.1007/s00122-013-2255-x

Habier, D., Fernando, R. L., Kizilkaya, K., and Garrick, D. J. (2011). Extension of the Bayesian alphabet for genomic selection. BMC Bioinformatics 12, 186. doi: 10.1186/1471-2105-12-186

Habyarimana, E. (2016). Genomic prediction for yield improvement and safeguarding of genetic diversity in CIMMYT spring wheat (Triticum aestivum L.). Aust. J. Crop Sci. 10, 127-136.

Hallauer, A. R., Carena, M. J., and Filho, J. B. M., (2010). Quantitative genetics in maize breeding. New York: Springer. doi: 10.1007/978-1-4419-0766-0_12

Herter, C. P., Ebmeyer, E., Kollers, S., Korzun, V., Würschum, T., and Miedaner, T. (2019). Accuracy of within- and among-family genomic prediction for Fusarium head blight and Septoria tritici blotch in winter wheat. Theor. Appl. Genet. 132, 1121-1135. doi: 10.1007/s00122-018-3264-6

Hickey, J. M., Chiurugwi, T., Mackay, I., Powell, W., Hickey, J. M., Chiurugwi, T., et al. (2017). Genomic prediction unifies animal and plant breeding programs to form platforms for biological discovery. Nat. Genet. 49, 1297-1303. doi: 10.1038/ng.3920 
Huang, X., Feng, Q., Qian, Q., Zhao, Q., Wang, L., Wang, A., et al. (2009). Highthroughput genotyping by whole-genome resequencing. Genome Res. 19, 1068-1076. doi: 10.1101/gr.089516.108

Huang, X., Wei, X., Sang, T., Zhao, Q., Feng, Q., Zhao, Y., et al. (2010). Genomewide association studies of 14 agronomic traits in rice landraces. Nat. Genet. 42 , 961-967. doi: 10.1038/ng.695

Huang, X., Yang, S., Gong, J., Zhao, Y., Feng, Q., Gong, H., et al. (2015). Genomic analysis of hybrid rice varieties reveals numerous superior alleles that contribute to heterosis. Nat. Commun. 6, 6258. doi: 10.1038/ncomms7258

Isidro, J., Jannink, J.-L., Akdemir, D., Poland, J., Heslot, N., and Sorrells, M. E. (2015). Training set optimization under population structure in genomic selection. Theor. Appl. Genet. 128, 145-158. doi: 10.1007/s00122-014-2418-4

Jiang, Y., and Reif, J. C. (2015). Modeling epistasis in genomic selection. Genetics 201, 759-768. doi: 10.1534/genetics.115.177907

Jonas, E., and de Koning, D.-J. (2013). Does genomic selection have a future in plant breeding? Trends Biotechnol. 31, 497-504. doi: 10.1016/j.tibtech.2013.06.003

Kemper, K. E., Bowman, P. J., Hayes, B. J., Visscher, P. M., and Goddard, M. E. (2018). A multi-trait Bayesian method for mapping QTL and genomic prediction. Genet. Sel. Evol. 50, 10. doi: 10.1186/s12711-018-0377-y

Lado, B., Barrios, P. G., Quincke, M., Silva, P., and Gutiérrez, L. (2016). Modeling genotype $\times$ environment interaction for genomic selection with unbalanced data from a wheat breeding program. Crop Sci. 56, 2165-2179. doi: 10.2135/ cropsci2015.04.0207

Li, H., Peng, Z., Yang, X., Wang, W., Fu, J., Wang, J., et al. (2013). Genome-wide association study dissects the genetic architecture of oil biosynthesis in maize kernels. Nat. Genet. 45, 43-50. doi: 10.1038/ng.2484

Lipka, A. E., Tian, F., Wang, Q., Peiffer, J., Li, M., Bradbury, P. J., et al. (2012). GAPIT: genome association and prediction integrated tool. Bioinformatics 28, 2397-2399. doi: 10.1093/bioinformatics/bts444

Liu, H., and Chen, G.-B. (2018). A new genomic prediction method with additivedominance effects in the least-squares framework. Heredity 121, 196-204. doi: 10.1038/s41437-018-0099-5

Liu, H., Yu, G., Wei, B., Wang, Y., Zhang, J., Hu, Y., et al. (2015). Identification and phylogenetic analysis of a novel starch synthase in maize. Front. Plant Sci. 6, 1013. doi: 10.3389/fpls.2015.01013

Liu, X., Wang, H., Wang, H., Guo, Z., Xu, X., Liu, J., et al. (2018). Factors affecting genomic selection revealed by empirical evidence in maize. Crop J. 6, 341-352. doi: 10.1016/j.cj.2018.03.005

Lopez-Cruz, M., Crossa, J., Bonnett, D., Dreisigacker, S., Poland, J., Jannink, J.-L., et al. (2015). Increased prediction accuracy in wheat breeding trials using a marker $\times$ environment interaction genomic selection model. G3 5, 569-582. doi: $10.1534 / \mathrm{g} 3.114 .016097$

Makarevitch, I., Thompson, A., Muehlbauer, G. J., and Springer, N. M. (2012). Brd 1 gene in maize encodes a brassinosteroid C-6 oxidase. PLoS One 7, e30798. doi: 10.1371/journal.pone.0030798

Mastrodomenico, A. T., Bohn, M. O., Lipka, A. E., and Below, F. E. (2019). Genomic selection using maize Ex-plant variety protection germplasm for the prediction of nitrogen-use traits. Crop Sci. 59, 212-220. doi: 10.2135/cropsci2018.06.0398

Melchinger, A., Geiger, H., Utz, H., and Schnell, F. (2003). Effect of recombination in the parent populations on the means and combining ability variances in hybrid populations of maize (Zea mays L.). Theor. Appl. Genet. 106, 332-340. doi: 10.1007/s00122-002-1000-7

Meuwissen, T. H. E., Hayes, B. J., and Goddard, M. E. (2001). Prediction of total genetic value using genome-wide dense marker maps. Genetics 157, 1819-1829.

Miedaner, T., Schulthess, A. W., Gowda, M., Reif, J. C., and Longin, C. F. H. (2017). High accuracy of predicting hybrid performance of Fusarium head blight resistance by mid-parent values in wheat. Theor. Appl. Genet. 130, 461-470. doi: 10.1007/s00122-016-2826-8

Morais Júnior, O. P., Batista Duarte, J., Breseghello, F., Coelho, A. S. G., Borba, T. C. O., Aguiar, J. T., et al. (2017). Relevance of additive and nonadditive genetic relatedness for genomic prediction in rice population under recurrent selection breeding. Genet. Mol. Res. 16, gmr16039849. doi: 10.4238/gmr16039849

Muñoz, P. R., Resende, M. F. R., Gezan, S. A., Resende, M. D. V., de los Campos, G., Kirst, M., et al. (2014). Unraveling additive from nonadditive effects using genomic relationship matrices. Genetics 198, 1759-1768. doi: 10.1534/ genetics.114.171322

Nirea, K. G., and Meuwissen, T. H. E. (2017). Improving production efficiency in the presence of genotype by environment interactions in pig genomic selection breeding programmes. J. Anim. Breed. Genet. 134, 119-128. doi: 10.1111/ jbg. 12250

Ober, U., Huang, W., Magwire, M., Schlather, M., Simianer, H., and Mackay, T. F. (2015). Accounting for genetic architecture improves sequence based genomic prediction for a Drosophila fitness trait. PLoS One 10, e0126880. doi: 10.1371/ journal.pone.0126880

Pan, Q., Xu, Y., Li, K., Peng, Y., Zhan, W., Li, W., et al. (2017). The genetic basis of plant architecture in 10 maize recombinant inbred line populations. Plant Physiol. 175, 858-873. doi: 10.1104/pp.17.00709

Peiffer, J. A., Romay, M. C., Gore, M. A., Flint-Garcia, S. A., Zhang, Z., Millard, M. J., et al. (2014). The genetic architecture of maize height. Genetics 196, 1337-1356. doi: 10.1534/genetics.113.159152

Pérez, P., and de los Campos, G. (2014). Genome-wide regression \& prediction with the BGLR statistical package. Genetics 198, 483-495. doi: 10.1534/genetics.114.164442

Plieschke, L., Edel, C., Pimentel, E. C., Emmerling, R., Bennewitz, J., and Götz, K.-U. (2015). A simple method to separate base population and segregation effects in genomic relationship matrices. Genet. Sel. Evol. 47, 53. doi: 10.1186/s12711-015-0130-8

Price, A. L., Patterson, N. J., Plenge, R. M., Weinblatt, M. E., Shadick, N. A., and Reich, D. (2006). Principal components analysis corrects for stratification in genome-wide association studies. Nat. Genet. 38, 904-909. doi: 10.1038/ng1847

Raoul, J., Swan, A. A., and Elsen, J.-M. (2017). Using a very low-density SNP panel for genomic selection in a breeding program for sheep. Genet. Sel. Evol. 49, 76. doi: 10.1186/s12711-017-0351-0

Rezende, F. M., Nani, J. P., and Peñagaricano, F. (2019). Genomic prediction of bull fertility in US Jersey dairy cattle. J. Dairy Sci. 102, 1-11. doi: 10.3168/ jds.2018-15810

Rice, B., and Lipka, A. E. (2019). Evaluation of RR-BLUP genomic selection models that incorporate peak genome-wide association study signals in maize and sorghum. Plant Genome 12, 1-14. doi: 10.3835/plantgenome2018.07.0052

Rio, S., Mary-Huard, T., Moreau, L., and Charcosset, A. (2019). Genomic selection efficiency and a priori estimation of accuracy in a structured dent maize panel. Theor. Appl. Genet. 132, 81-96. doi: 10.1007/s00122-018-3196-1

Robledo, D., Matika, O., Hamilton, A., and Houston, R. D. (2018). Genome-wide association and genomic selection for resistance to amoebic gill disease in Atlantic salmon. G3 8, 1195-1203. doi: 10.1534/g3.118.200075

Sarinelli, J. M., Murphy, J. P., Tyagi, P., Holland, J. B., Johnson, J. W., Mergoum, M., et al. (2019). Training population selection and use of fixed effects to optimize genomic predictions in a historical USA winter wheat panel. Theor. Appl. Genet. 132, 1-15. doi: 10.1007/s00122-019-03276-6

Schopp, P., Müller, D., Technow, F., and Melchinger, A. E. (2017). Accuracy of genomic prediction in synthetic populations depending on the number of parents, relatedness, and ancestral linkage disequilibrium. Genetics 205, 441454. doi: 10.1534/genetics.116.193243

Slavov, G. T., Nipper, R., Robson, P., Farrar, K., Allison, G. G., Bosch, M., et al. (2014). Genome-wide association studies and prediction of 17 traits related to phenology, biomass and cell wall composition in the energy grass Miscanthus sinensis. New Phytol. 201, 1227-1239. doi: 10.1111/nph.12621

Spindel, J., Begum, H., Akdemir, D., Virk, P., Collard, B., Redoña, E., et al. (2015). Genomic selection and association mapping in rice (Oryza sativa): effect of trait genetic architecture, training population composition, marker number and statistical model on accuracy of rice genomic selection in elite, tropical rice breeding lines. PLoS Genet. 11, e1004982. doi: 10.1371/journal.pgen.1004982

Spindel, J. E., Begum, H., Akdemir, D., Collard, B., Redoña, E., Jannink, J.-L., et al. (2016). Genome-wide prediction models that incorporate de novo GWAS are a powerful new tool for tropical rice improvement. Heredity 116, 395-408. doi: 10.1038/hdy.2015.113

Su, G., Christensen, O. F., Ostersen, T., Henryon, M., and Lund, M. S. (2012). Estimating additive and non-additive genetic variances and predicting genetic merits using genome-wide dense single nucleotide polymorphism markers. PLoS One 7, e45293. doi: 10.1371/journal.pone.0045293

Su, G., Christensen, O. F., Janss, L., and Lund, M. S. (2014). Comparison of genomic predictions using genomic relationship matrices built with different weighting factors to account for locus-specific variances. J. Dairy Sci. 97, 6547-6559. doi: 10.3168 /jds.2014-8210

Suzuki, M., Settles, A. M., Tseung, C.-W., Li, Q.-B., Latshaw, S., Wu, S., et al. (2006). The maize viviparous15 locus encodes the molybdopterin synthase small subunit. Plant J. 45, 264-274. doi: 10.1111/j.1365-313X.2005.02620.x 
Taylor, J., and Butler, D. (2017). R package ASMap: efficient genetic linkage map construction and diagnosis. J. Stat. Softw. 79, 1-28. doi: 10.18637/jss.v079.i06

Technow, F., Bürger, A., and Melchinger, A. E. (2013). Genomic prediction of northern corn leaf blight resistance in maize with combined or separated training sets for heterotic groups. G3 3, 197-203. doi: 10.1534/g3.112.004630

Toosi, A., Fernando, R. L., and Dekkers, J. C. M. (2010). Genomic selection in admixed and crossbred populations. J. Anim. Sci. 88, 32-46. doi: 10.2527/ jas.2009-1975

VanRaden, P. M. (2008). Efficient methods to compute genomic predictions. J. Dairy Sci. 91, 4414-4423. doi: 10.3168/jds.2007-0980

Varona, L., Legarra, A., Toro, M. A., and Vitezica, Z. G. (2018). Non-additive effects in genomic selection. Front. Genet. 9, 78. doi: 10.3389/fgene.2018.00078

Viret, J. F., Schantz, M. L., and Schantz, R. (1993). A maize cDNA encoding a type II chlorophyll a/b-binding protein of photosystem II. Plant Physiol. 102, 13611362. doi: 10.1104/pp.102.4.1361

Wang, X., Wang, H., Liu, S., Ferjani, A., Li, J., Yan, J., et al. (2016). Genetic variation in ZmVPP1 contributes to drought tolerance in maize seedlings. Nat. Genet. 48, 1233-1241. doi: 10.1038/ng.3636

Wang, X., Li, L., Yang, Z., Zheng, X., Yu, S., Xu, C., et al. (2017). Predicting rice hybrid performance using univariate and multivariate GBLUP models based on North Carolina mating design II. Heredity 118, 302-310. doi: 10.1038/ hdy.2016.87

Wang, X., Xu, Y., Hu, Z., and Xu, C. (2018). Genomic selection methods for crop improvement: current status and prospects. Crop J. 6, 330-340. doi: 10.1016/j. cj.2018.03.001

Werner, C. R., Qian, L., Voss-Fels, K. P., Abbadi, A., Leckband, G., Frisch, M., et al. (2018). Genome-wide regression models considering general and specific combining ability predict hybrid performance in oilseed rape with similar accuracy regardless of trait architecture. Theor. Appl. Genet. 131, 299-317. doi: 10.1007/s00122-017-3002-5

Whittaker, J. C., Thompson, R., and Denham, M. C. (2000). Marker-assisted selection using ridge regression. Genet. Res. 75, 249-252. doi: 10.1017/ S0016672399004462

Xiao, Y., Tong, H., Yang, X., Xu, S., Pan, Q., Qiao, F., et al. (2016). Genome-wide dissection of the maize ear genetic architecture using multiple populations. New Phytol. 210, 1095-1106. doi: 10.1111/nph.13814

Xu, C., Ren, Y., Jian, Y., Guo, Z., Zhang, Y., Xie, C., et al. (2017a). Development of a maize $55 \mathrm{~K}$ SNP array with improved genome coverage for molecular breeding. Mol. Breed. 37, 20. doi: 10.1007/s11032-017-0622-Z

Xu, D., Wang, X., Huang, C., Xu, G., Liang, Y., Chen, Q., et al. (2017b). Glossy15 plays an important role in the divergence of the vegetative transition between maize and its progenitor, teosinte. Mol. Plant 10, 1579-1583. doi: 10.1016/j. molp.2017.09.016

$\mathrm{Xu}, \mathrm{S}$., Zhu, D., and Zhang, Q. (2014). Predicting hybrid performance in rice using genomic best linear unbiased prediction. P. Natl. Acad. Sci. 111, 12456-12461. doi: 10.1073/pnas.1413750111

Xu, S., Xu, Y., Gong, L., and Zhang, Q. (2016). Metabolomic prediction of yield in hybrid rice. Plant J. 88, 219-227. doi: 10.1111/tpj.13242

Xu, Y., Wang, X., Ding, X., Zheng, X., Yang, Z., Xu, C., et al. (2018). Genomic selection of agronomic traits in hybrid rice using an NCII population. Rice 11, 32. doi: 10.1186/s12284-018-0223-4
Yang, J., Mezmouk, S., Baumgarten, A., Buckler, E. S., Guill, K. E., McMullen, M. D., et al. (2017). Incomplete dominance of deleterious alleles contributes substantially to trait variation and heterosis in maize. PLoS Genet. 13, e1007019. doi: 10.1371/journal.pgen.1007019

Yu, J., Pressoir, G., Briggs, W. H., Vroh Bi, I., Yamasaki, M., Doebley, J. F., et al. (2006). A unified mixed-model method for association mapping that accounts for multiple levels of relatedness. Nat. Genet. 38, 203-208. doi: 10.1038/ng1702

Yuan, Y., Cairns, J. E., Babu, R., Gowda, M., Makumbi, D., Magorokosho, C., et al. (2019). Genome-wide association mapping and genomic prediction analyses reveal the genetic architecture of grain yield and flowering time under drought and heat stress conditions in maize. Front. Plant Sci. 9, 1919. doi: 10.3389/ fpls.2018.01919

Zhang, A., Wang, H., Beyene, Y., Semagn, K., Liu, Y., Cao, S., et al. (2017a). Effect of trait heritability, training population size and marker density on genomic prediction accuracy estimation in 22 bi-parental tropical maize populations. Front. Plant Sci. 8, 1916. doi: 10.3389/fpls.2017.01916

Zhang, H., Yin, L., Wang, M., Yuan, X., and Liu, X. (2019). Factors affecting the accuracy of genomic selection for agricultural economic traits in maize, cattle, and pig populations. Front. Genet. 10, 189. doi: 10.3389/fgene.2019.00189

Zhang, X., Pérez-Rodríguez, P., Burgueño, J., Olsen, M., Buckler, E., Atlin, G., et al. (2017b). Rapid cycling genomic selection in a multi-parental tropical maize population. G3 7, 2315-2326. doi: 10.1534/g3.117.043141

Zhang, X., Huang, C., Wu, D., Qiao, F., Li, W., Duan, L., et al. (2017c). Highthroughput phenotyping and QTL mapping reveals the genetic architecture of maize plant growth. Plant Physiol. 176, 1554-1564. doi: 10.1104/pp.16.01516

Zhang, Z., Erbe, M., He, J., Ober, U., Gao, N., Zhang, H., et al. (2015). Accuracy of whole-genome prediction using a genetic architecture-enhanced variancecovariance matrix. G3 5, 615-627. doi: 10.1534/g3.114.016261

Zhang, Z., Ober, U., Erbe, M., Zhang, H., Gao, N., He, J., et al. (2014). Improving the accuracy of whole genome prediction for complex traits using the results of genome wide association studies. PloS One 9, e93017. doi: 10.1371/journal. pone.0093017

Zhao, Y., Li, Z., Liu, G., Jiang, Y., Maurer, H. P., Würschum, T., et al. (2015). Genome-based establishment of a high-yielding heterotic pattern for hybrid wheat breeding. P. Natl. Acad. Sci. 112, 15624-15629. doi: 10.1073/ pnas. 1514547112

Zhou, Z., Zhang, C., Zhou, Y., Hao, Z., Wang, Z., Zeng, X., et al. (2016). Genetic dissection of maize plant architecture with an ultra-high density bin map based on recombinant inbred lines. BMC Genomics 17, 178. doi: 10.1186/ s12864-016-2555-z

Conflict of Interest Statement: The authors declare that the research was conducted in the absence of any commercial or financial relationships that could be construed as a potential conflict of interest.

Copyright (C) $2019 \mathrm{Liu}$, Wang, Hu, Li, Liu, Wu and Huang. This is an open-access article distributed under the terms of the Creative Commons Attribution License (CC $B Y)$. The use, distribution or reproduction in other forums is permitted, provided the original author(s) and the copyright owner(s) are credited and that the original publication in this journal is cited, in accordance with accepted academic practice. No use, distribution or reproduction is permitted which does not comply with these terms. 